\title{
Upregulated CTHRC1 promotes human epithelial ovarian cancer invasion through activating EGFR signaling
}

\author{
JUN YE $^{1 *}$, WEI CHEN ${ }^{1 *}$, ZHI-YONG WU ${ }^{2}$, JIN-HUI ZHANG ${ }^{1}$, HE FEI $^{1}$, LI-WEN ZHANG $^{1}$, \\ YA-HUI WANG ${ }^{3}$, YA-PING CHEN $^{1}$ and XIAO-MEI YANG ${ }^{3}$
}

\begin{abstract}
${ }^{1}$ The Fifth People's Hospital of Shanghai, Shanghai 200240; ${ }^{2}$ Department of Gynecology, Obstetrics and Gynecology Hospital, Fudan University, Shanghai 200011; ${ }^{3}$ State Key Laboratory of Oncogenes and Related Genes, Shanghai Cancer Institute, Renji Hospital, Shanghai Jiao Tong University School of Medicine, Shanghai 200240, P.R. China
\end{abstract}

Received May 14, 2016; Accepted October 17, 2016

DOI: $10.3892 /$ or.2016.5198

\begin{abstract}
Epithelial ovarian cancer (EOC) is the major cause of deaths from gynecologic malignancies, and metastasis is the main cause of cancer related death. Collagen triple helix repeat containing-1 (CTHRC1) is a secreted protein that has the ability to inhibit collagen matrix synthesis. In this study, we found that high CTHRC1 expression was associated with poor prognosis of EOC. In vitro experiments showed that CTHRC1 promoted migration and invasion of ovarian cancer cells. CTHRC1 had no effect on ovarian cancer cells viability. Additionally, EGFR inhibitors reduced the promotion effects of CTHRC1 on EOC cell invasion. After silencing of CTHRC1, downregulated expression of phosphorylation of EGFR/ERK1/2/AKT was observed in ovarian cancer cells. Taken together, our results suggest a role for CTHRC1 in the progression of ovarian cancer and identified CTHRC1 as a potentially important predictor for human ovarian cancer prognosis.
\end{abstract}

\section{Introduction}

Ovarian cancer is the major cause of deaths from gynecologic malignancies and the 5th leading cause of cancer-related deaths among women in the United States. According to the

Correspondence to: Dr Ya-Ping Chen, The Fifth People's Hospital of Shanghai, Fudan University, 108 He Qing Road, Min Hang, Shanghai 200240, P.R. China

E-mail: chenyaping@5thhospital.com

Dr Xiao-Mei Yang, State Key Laboratory of Oncogenes and Related Genes, Shanghai Cancer Institute, Renji Hospital, Shanghai Jiao Tong University School of Medicine, 800 Dongchuan Road, Shanghai 200240, P.R. China

E-mail: xmyang@sibs.ac.cn

*Contributed equally

Key words: epithelial ovarian cancer, collagen triple helix repeat containing-1, EGFR, ERK1/2, AKT
National Cancer Institute (NCI) report prediction, 21,290 new cases would be diagnosed with ovarian cancer in America in 2015, and 14,180 patients would die of this disease (1).

Most cancer-related deaths result from the formation of metastases (2), which are difficult to detect and can induce relapse for years after treatment of the primary tumor (3). Ovarian cancer patients die of their disease mainly attributed to metastasis (4). Unfortunately, the mechanism of metastasis is still unclear.

Collagen triple helix repeat containing-1 (CTHRC1) is a $30-\mathrm{kDa}$ secreted protein that has the ability to inhibit collagen matrix synthesis. It is highly expressed in cartilage, developing bones, and myofibroblasts during skin wound healing (5). A human tumor complementary DNA array analysis has shown that the CTHRC1 gene is expressed in the majority of human solid cancers (6). Recent studies reported that CTHRC1 promoted tumor cell metastasis by different signaling pathways in different cancers. In gastric cancer, CTHRC1 has been shown to be upregulated by promoter demethylation and in response to TGF- $\beta$ signaling (7), while in human non-small cell lung cancer, CTHRC1 mediates aggressiveness via GSK-3 $\beta / \beta$-catenin pathway (8).

Epidermal growth factor receptor (EGFR) has received considerable attention in ovarian cancer research, because up to $75 \%$ of primary epithelian ovarian cancers (EOC) overexpress EGFR (9). Overexpression of EGFR is associated with advanced-stage disease and poor prognosis (10). Furthermore, EGFR and its family members are the major contributors of a complex signaling cascade that modulates growth, signaling, differentiation, adhesion, migration and survival of cancer cells (11). Published data demonstrates that EGFR plays an important role in tumor progression (12). Thus EGFR is considered as a critical molecular target for therapy in advanced ovarian cancer.

EGFR is closely related to ovarian cancer metastasis (13) and a number of cellular signals such as protein kinase (AKT) and extracellular signal-regulated kinase1/2 (ERK1/2) (14-16) can be activated due to EGFR activation. Although recent study showed CTHRC1 may promote EOC metastasis through Wnt/catinen signal pathway (17), but little is known about whether CTHRC1 was involved in EGFR signaling to modulate EOC cell metastasis. 
Zhou et al reported that microRNA-7 inhibited tumor metastasis and reversed EMT through AKT and ERK1/2 pathway inactivation by reducing EGFR expression in EOC cell lines (18), this led us to investigate whether the EGFR and its downstream signaling pathways were involved in the effect on ovarian cancer cell migration and invasion induced by CTHCR1.

In this study, we examined the CTHRC1 expression in EOC and found that CTHRC1 played an important role in ovarian cancer cell metastasis. EGFR inhibitors can block recombinant CTHRC1 induced promotion of ovarian cancer cell migration and invasion. Silencing of CTHRC1 with siRNA blocked phosphorylation of EGFR, ERK1/2 and Akt in the ES-2 cell line. We demonstrated that CTHRC1 may promote migration and invasion of ovarian cancer cells through activated signaling of EGFR/ERK1/2/AKT.

\section{Materials and methods}

Cell culture. Human ovarian cancer cell line SKOV3, ES2, CAOV3, HEY, COV318 were obtained as gifts from Shanghai Cancer Institute. Cells were cultured in Dulbecco's modified Eagle's medium (DMEM; Invitrogen, USA) supplemented with $10 \%$ (v/v) fetal bovine serum (Invitrogen), $100 \mathrm{U} / \mathrm{ml}$ penicillin and $100 \mu \mathrm{g} / \mathrm{ml}$ streptomycin, at $37^{\circ} \mathrm{C}$ in an incubator with $5 \% \mathrm{CO}_{2}$ condition.

Clinical samples. The studied human ovary tissue microarray contained 83 cases of ovarian cancer. These tissues were obtained from Department of Gynecology, Obstetrics and Gynecology Hospital, Fudan University from 2006 to 2009. None of them had received chemotherapy, radiotherapy and other related antitumor therapies before surgery. Eighteen cases of normal ovarian tissues were selected as the control group. All human ovary tissues were obtained with informed consent and all protocols were approved by the ethics review committee of the World Health Organization Collaborating Center for Research in Human Production.

Tissue microarray construction. Human ovary tissue microarray was constructed by Suzhou Xinxin Biotechnology Co. Ltd. (Xinxin Biotechnology Co., Suzhou, China). Two 1.6-mm cores per donor block were transferred into a recipient block tissue microarray. Three-micron thick sections were cut from the recipient block and transferred to glass slides with an adhesive tape transfer system for ultraviolet cross linkage.

Immunohistochemical staining. The tissue microarray glass slides were baked at $55^{\circ} \mathrm{C}$ for $1 \mathrm{~h}$, and then de-paraffinized gradually through xylene, $50 \%$ xylene, gradient concentrations of ethanol until immersed in tap water. Tissue sections were blocked for peroxidase activity with $0.3 \%$ hydrogen peroxide at $37^{\circ} \mathrm{C}$ for $30 \mathrm{~min}$. Antigen retrieval was carried out by boiling in $10 \mathrm{mmol} / 1$ citrate buffer ( $\mathrm{pH} 6.0$ ) for $15 \mathrm{~min}$. Then the tissues were incubated with CTHRC1 antibody (rabbit, 1:200 dilution, Abcam Biotechnology) overnight at $4^{\circ} \mathrm{C}$. The next day, the tissues were washed with phosphate buffer solution (PBS) three times and incubated with HRP-labeled anti-rabbit secondary antibody (1:200 dilution, Dako, Carpinteria, CA, USA) for $1 \mathrm{~h}$ at room temperature. Immunostaining was carried out using diaminobenzidine substrate chromogen method. After immunostaining tissues were immersed into hematoxylin for nuclear staining. The TMA slides were then dehydrated through gradient concentrations of ethanol, cleared with xylene, and coverslipped with neutral balsam. Five high-power fields were randomly chosen, and $\geq 300$ cells were counted per field. Expression score was determined by staining intensity and immunoreactive cell percentage. Scoring was conducted according to the ratio and intensity of positivestaining cells: $0-5 \%$ scored $0 ; 6-35 \%$ scored $1 ; 36-70 \%$ scored 2 ; $>70 \%$ scored 3 . The final score of CTHCR1 expression was designated as low or high expression group as follows: low expression, score 0-1; high expression, score 2-3. All the scores of CTHRC1 expression were performed in a blinded manner and determined independently by two senior pathologists.

RNA interference-based gene silencing experiment. Stably transfected clones were selected using ES2 cells, and transfection was performed with SuperFectin according to the manufacturer's instructions. SiRNA interference plasmid was purchased from GenePharma and designed to target the following cDNA sequences: CTHRC1-shRNA1, sense, 5'-CAG CGU UGG UAU UUC ACA UUT-3'; antisense, 5'-AUG UGA AAU ACC AAC GCU GTT-3'. CTHRC1shRNA2, sense, 5'-GCU UCU ACU GGA UGG AAU UTT-3'; antisense, 5'-AAU UCC AUC CAG UAG AAG CTT-3'. CTHRC1-negative control: sense, 5'-UUC UCC GAA CGU GUC ACG UTT-3'; antisense, 5'-ACG UGA CAC GUU CGG AGA ATT-3'. Stable shRNA-expressing clones were selected with $2 \mu \mathrm{g} / \mathrm{ml}$ puromycin (Sangon, Shanghai, China) and the silencing effects were verified by qRT-PCR and western blot analysis.

Western blot analysis. Cells were lysed in lysis buffer $(50 \mathrm{mM}$ Tris- $\mathrm{HCl}, 150 \mathrm{mM} \mathrm{NaCl}, 1 \%$ Triton-X 100, 1 mM each $\mathrm{MgCl}_{2}$, $\mathrm{MnCl}_{2}$ and $\mathrm{CaCl}_{2}, 1 \mathrm{mM}$ PMSF and $10 \mathrm{mM}$ sodium fluoride) and protease inhibitor cocktail. Proteins were separated by SDS-PAGE under reducing condition, followed by immunoblotting with specific primary antibodies and species-specific secondary antibodies. Bound secondary antibodies were revealed by Odyssey imaging system (LI-COR Biosciences, Lincoln, NE, USA). Quantification was analyzed using ImageJ software. The antibodies used in this study included: antiCTHRC1 (rabbit, 1:1,000, Abcam Biotechnology), anti-EGFR and anti-pEGFR (Tyr1148) (rabbit, 1:1,000, Cell Signaling Technology), anti-AKT and anti-pAKT (S473) (rabbit, 1:1,000, Cell Signaling Technology), anti-ERK1/2 and anti-pERK1/2 (Thr202/Tyr204) (rabbit, 1:1,000, Cell Signaling Technology), anti- $\beta$-actin (rabbit, 1:1,000, Sigma Biotechnology).

Quantitative real-time PCR. Total cellular RNA was extracted using TRIzol reagent (Takara) and reversely transcribed through PrimeScript RT-PCR kit (Takara) according to the protocol. The CTHRC1 mRNA expression was determined by real-time PCR using SYBR Premix Ex Taq (Takara) on a 7500 real-time PCR system (Applied Biosystems) at the following cycling settings: one initial cycle at $95^{\circ} \mathrm{C}$ for $10 \mathrm{sec}$ followed by 40 cycles of $5 \mathrm{sec}$ at $95^{\circ} \mathrm{C}$ and $31 \mathrm{sec}$ at $60^{\circ} \mathrm{C}$. Primers were CTHRC1: (sense) 5'-TGG ACA CCC AAC TAC AAG CA-3' and (antisense) 5'-GAA CAA GTG CCA ACC CAG 
A Normal ovarian tissue

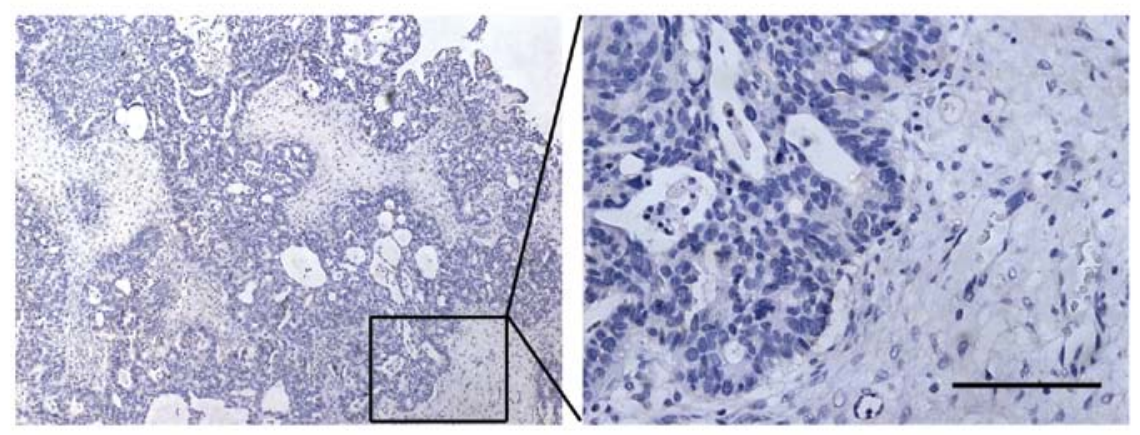

B

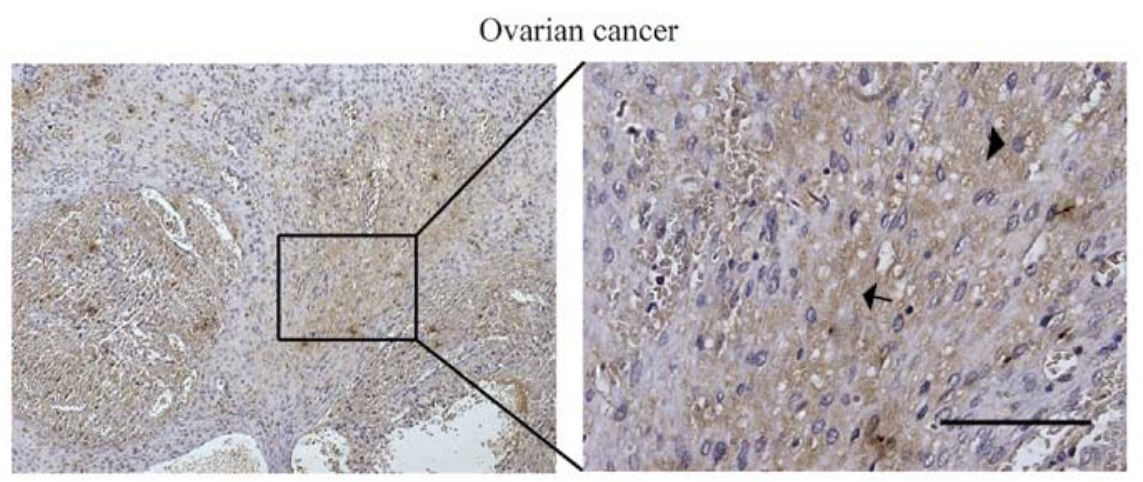

Figure 1. Immunohistochemical staining reveals CTHRC1 expression in paraffin-embedded ovarian cancer tissues. (A) Normal ovarian tissues showed low CTHRC1 expression. (B) Cancer tissues showed high CTHRC1 expression. The photos on the right are magnified images from the boxed region of the left image. Arrowhead, intracellular staining; arrow, extracellular staining. Scale bar, $100 \mu \mathrm{m}$.

AT-3'. 18S (primers: sense 5'-TGC GAG TAC TCA ACA CCA ACA-3', antisense 5'-GCA TAT CTT CGG CCC ACA-3') was used as an internal reference.

Cell viability assay (CCK8 assay). Cell viability was detected using a standard Cell Counting Kit- 8 assay. Ovarian cancer cells were plated in 96-well plates at a density of $3 \times 10^{4}$ cells per well with $100 \mu \mathrm{l}$ of complete culture medium. We added $10 \mu \mathrm{l}$ of reagent from Cell Counting Kit-8 (Dojindo, Kumamoto, Japan) to each well for detection at days 1, 2, 3, 4 and 5 . After $1 \mathrm{~h}$ of incubation at $37^{\circ} \mathrm{C}$, the optical density was measured using microplate reader at a wavelength of $450 \mathrm{~nm}$. The experiment was repeated three times.

In vitro migration and invasion assays. For the in vitro Transwell migration assay, $4 \times 10^{4}$ ovarian cancer cells were suspended in DMEM medium and added to the upper chamber of each insert with the non-coated membrane. In the lower chamber $700 \mu 1$ DMEM medium with $5 \%$ FBS was added. After incubation for $24 \mathrm{~h}$ at $37^{\circ} \mathrm{C}, 5 \% \mathrm{CO}_{2}$, cells that migrated through the membrane filter were fixed and stained with crystal violet. The number of migrating cells was counted and imaged through an IX71 inverted microscope (Olympus Corp., Tokyo, Japan). Transwell invasion assay was carried out by adding $100 \mu \mathrm{l}$ Matrigel (BD Bioscience, Franklin Lakes, NJ, USA) into the upper chamber of the Transwell and placing $6 \times 10^{4}$ cells onto the Matrigel; $48 \mathrm{~h}$ later, cells that invaded through the membrane filter were fixed, stained with crystal violet, counted and imaged. At least five grids per field were counted. All assays were independently repeated three times.

Agent. AG1478; erlotinib (EGFR inhibitor, effective dose: $100 \mathrm{nmol} / 1$, MedChem Express, USA). CTHRC1 recombinant protein (rCTHRC1) was obtained as a gift from Shanghai Cancer Institute.

Statistical analysis. The data were analyzed as the mean \pm standard deviation (SD). Statistical analyses were conducted using SPSS 14.0 software (SPSS Inc). We performed Pearson's test in cross tables to assess the relationships between expression levels of CTHRC1 and clinicopathological factors. Overall survival (OS) was calculated using Kaplan-Meier method. Student's t-test has been performed for calculating statistical significance between groups. Difference in P-value $<0.05$ is referred to as statistically significant. All statistical tests were two-sided.

\section{Results}

CTHRCl protein expression level is excessively elevated in epithelial ovarian cancer tissues. We performed an immunohistochemical (IHC) analysis of a tissue microarray that contained 83 epithelial ovarian cancer (EOC) tissue samples and 18 normal ovarian tissues. As shown in Fig. 1, the result showed strong CTHRC1 staining was detected in the majority of EOC samples $(55.4 \%, 46 / 83)$ while rarely detected in the normal ovarian tissues $(11.1 \%, 2 / 18)$. CTHRC1 was detected 


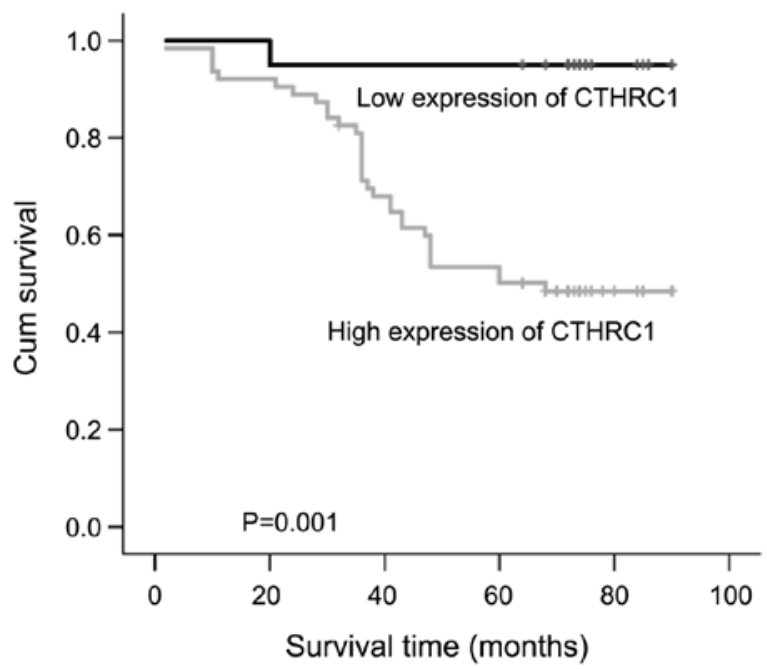

Figure 2. Cumulative survival curves according to CTHRC1 expression. On the basis of score of CTHRC1, patients were divided into high CTHRC1 (score $\geq 2$ ) and low CTHRC1 (score $<2$ ). Patients in the high-expression CTHRC1 group had significantly shortened overall survival.

Table I. Correlation between CTHRC1 expression and clinicopathologic parameters in 83 ovarian cancer patients.

\begin{tabular}{lrrrr}
\hline & & \multicolumn{2}{c}{ CTHRC1 } & \\
\cline { 3 - 4 } Parameters & Total & $\begin{array}{c}\text { Score }<2, \\
\mathrm{n}(\%)\end{array}$ & $\begin{array}{c}\text { Score } \geq 2, \\
\mathrm{n}(\%)\end{array}$ & P-value \\
\hline Ovarian cancer group & 83 & $38(45.6)$ & $46(55.4)$ & $\mathbf{0 . 0 0 0}^{\mathrm{a}}$ \\
Control group & 18 & $16(88.9)$ & $2(11.1)$ & \\
Histologic subgroups & & & & \\
$\quad$ Serous & 60 & $12(20.0)$ & $48(80.0)$ & 0.104 \\
$\quad$ Endometrioid & 11 & $5(45.4)$ & $6(54.6)$ & \\
$\quad$ Clear cell & 12 & $3(25.0)$ & $9(75.0)$ & \\
FIGO stage & & & & \\
$\quad$ I-II & 39 & $17(43.6)$ & $22(56.4)$ & $\mathbf{0 . 0 0 0}^{\mathbf{a}}$ \\
$\quad$ III-IV & 44 & $3(6.8)$ & $41(93.2)$ & \\
Lymph node status & & & & \\
$\quad$ Negative & 23 & $10(43.4)$ & $13(56.6)$ & $\mathbf{0 . 0 0 0}^{\mathbf{a}}$ \\
$\quad$ Positive & 60 & $7(11.6)$ & $51(89.4)$ & \\
Tumor size (cm) & & & & \\
$\quad<2$ & 32 & $15(46.8)$ & $16(53.2)$ & $\mathbf{0 . 0 0 0}^{\mathbf{a}}$ \\
$\geq 2$ & 51 & $11(21.5)$ & $40(78.6)$ & \\
\hline
\end{tabular}

Statistical analyses were performed by Pearson $\chi^{2}$ test; ${ }^{\text {aThe P-value }}$ in bold emphasized statistical significance $(\mathrm{P}<0.001)$.

both as intracellular and extracellular expression of the ovarian cancer cell as marked by arrowhead and arrow in the images. Using the Kaplan-Meier analysis, we found that patients with higher expression of CTHRC1 were significantly associated with shorter overall survival (Fig. 2).
We further analyzed the relevance of CTHRC1 expression with patient clinicopathological parameters and found that high expression of CTHRC1 was closely related with adverse clinicopathological parameters of EOC, including FIGO stage, lymph node status and tumor size. No correlation with histologic subgroups of epithelian ovarian cancer was found (Table I).

The expression of CTHRC1 in ES2 cells transfected with LentishCTHRCl. To determine the optimal cell lines for further study, we measured the expression of CTHRC1 in five ovarian cell lines (HEY, CAOV3, ES2, COV318 and SKOV3). Our data showed that the expression of CTHRC1 was the highest in the ES2 cell line (Fig. 3A and B), an EOC cell line with highly metastatic potential (17). Therefore, ES2 cells were transfected with Lenti-shCTHRC1-(1,2), designated as sh1 and sh2, or a mock vector, which was labeled as control. The silencing effects of the Lenti-shRNAs in the cell line were validated by RT-PCR and western blotting (Fig. 3C-E). The results showed that CTHRC1 expression levels were significantly decreased by Lenti-shCTHRC1- $(1,2)$.

Silencing of CTHRCl or rCTHRC1 has no effects on ovarian cancer cell proliferation in vitro. To explore the effect of CTHRC1 on ovarian cancer cell growth, ES2 transfected with sh1 and sh2 were used to detect cell proliferation by Cell Counting Kit-8 (CCK8) assay. The results showed that silencing of CTHRC1 could not suppress the proliferation of the ES2 cells (Fig. 4A).

Next we set out to investigate the effect of purified recombinant CTHRC1 (rCTHRC1) on EOC cell viability. SKOV3 cells were seeded into 96-well plates at the same density and treated with gradient doses of CTHRC1. Compared to the control group, SKOV3 cell proliferation was not obviously enhanced by rCTHRC1 protein (Fig. 4B), which was consistent with the effect of shRNA interference.

Silencing of CTHRC1 suppresses ovarian cancer cell invasion and migration in vitro. To further investigate the functional role of CTHRC1 in ovarian cancer, we examined the effects of CTHRC1 on ovarian cancer cell migration and invasion in vitro. Compared to the control group, silencing of CTHRC1 significantly inhibited ES2 cell migration and invasion in vitro (Fig. 5A and B). These data indicate that CTHRC1 has a potent role in EOC cancer metastasis.

rCTHRCl promotes ovarian cancer cell invasion and migration in vitro. As a secreted protein, we explored the biological functions of CTHRC1, so the rCTHRC1 protein was applied to SKOV3 cells in a migration and Matrigel invasion assay. Gradient doses of 0, 20, 50 and $100 \mathrm{nM}$ rCTHRC1, respectively, were added into the lower chamber and cells were added in the upper chamber. Compared to the control group, SKOV3 cell migration and invasion were significantly enhanced by rCTHRC1 protein at doses of 50 and $100 \mathrm{nM}$ (Fig. 5C and D). At a dose of $50 \mathrm{nM}$, the promotion effect of cell motility by the $\mathrm{rCTHRC1}$ protein was the strongest. Our data indicate that CTHRC1 contributes greatly to the development of ovarian cancer metastasis.

EGFR inhibitors suppress ovarian cancer cell invasion and migration induced by rCTHRCl in vitro. Previous studies 

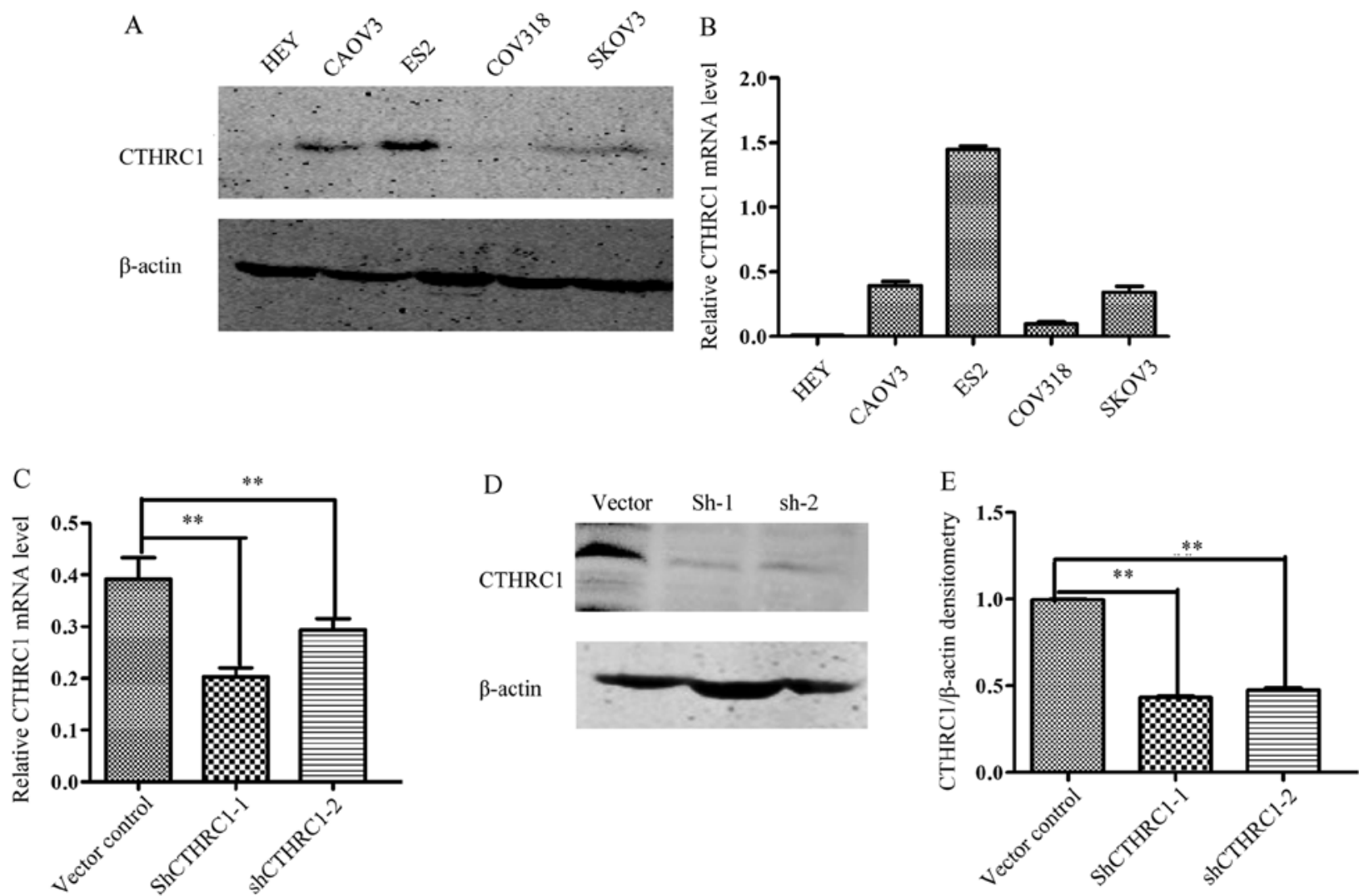

Figure 3. (A) Protein expression of CTHRC1 in five ovarian cancer cell lines. (B) mRNA expression of CTHRC1 in five ovarian cancer cell lines. (C) The expression level of CTHRC1 in ES2 cells transfected with Lenti-shCTHRC1-(1,2) or control vector was analyzed by qRT-PCR. The data are mean \pm SD ${ }^{* *} \mathrm{P}<0.01$, compared with control. (D) The protein expression of CTHRC1 in ES2 cells transfected with Lenti-shCTHRC1- $(1,2)$ or control vector was analyzed by western blotting. (E) The bar chart demonstrates the ratio of CTHRC1 protein level to $\beta$-actin level from (D) by densitometry.

A
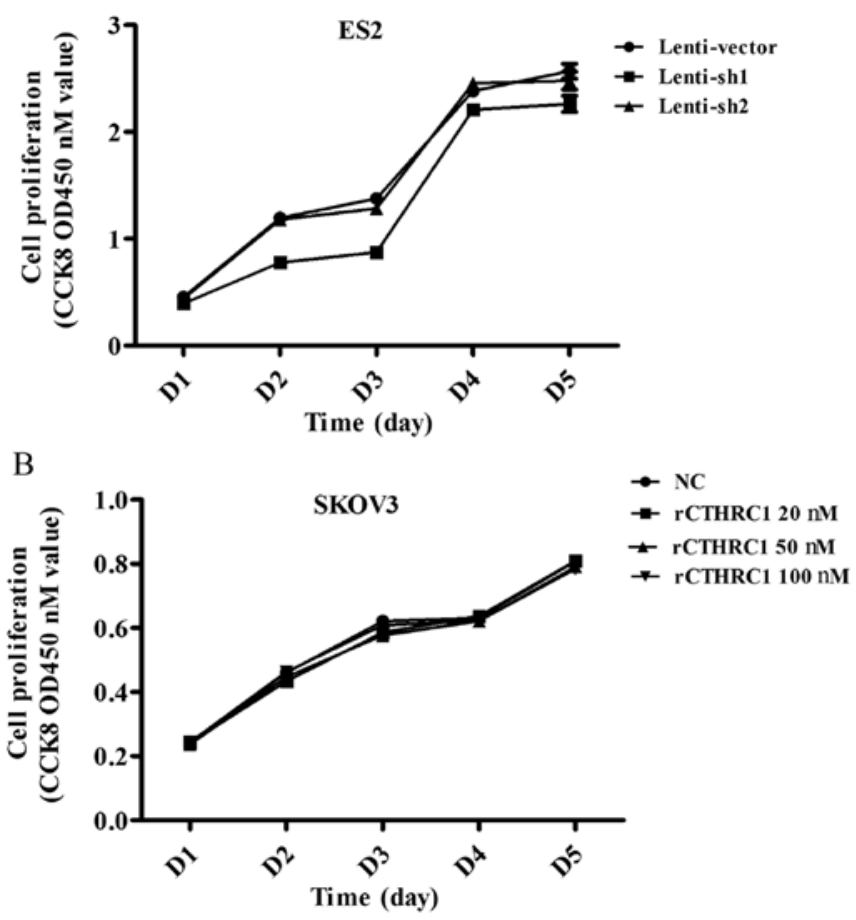

Figure 4. CTHRC1 has no effects on ovarian cancer cell proliferation in vitro. (A) ES2 cells transfected with Lenti-shCTHRC1 had no effect on cell proliferation. (B) rCTHRC1 did not promote SKOV3 cell proliferation. Values are means $\pm \mathrm{SD}, \mathrm{P}>0.05$. have demonstrated clear effects of EGFR signaling on ovarian cancer cell invasion $(13,19)$, but whether CTHRC1 regulate EGFR signaling in ovarian cancer cells has not been reported. Thus, we further determined EGFR signaling in CTHRC1 induced ovarian cancer cell migration and invasion. AG1478 and erlotinib, two inhibitors of EGFR signaling, were applied in the Transwell assay. The results indicated that AG1478 or erlotinib partially reversed rCTHRC1 induced cell migration and invasion (Fig. 5E and F). This result demonstrates that CTHRC1 promotes EOC cell migration and invasion through EGFR signaling.

CTHRC1 activates EGFR/EKR1/2/AKT signaling in ovarian cancer cells. We further investigated whether EGFR signaling was affected by CTHRC1. We found that the level of phosphorylated EGFR was clearly downregulated after CTHRC1 knockdown in ES2 cells (Fig. 6A). Existing data indicated that the effects of EGFR signaling on cell migration and invasion are mediated by PI3K/AKT and ERK1/2 signaling pathways in EOC $(14,20)$, therefore, we next investigated whether ERK1/2 and AKT were also involved in EGFR signal induced by CTHRC1 in EOC. We explored the pERK1/2 and pAKT expression after CTHRC1 knockdown in ES2 cells. The results showed that the expression of pEKR $1 / 2$ and pAKT was markedly decreased by CTHRC1 knockdown (Fig. 6B and C). We also detected ERK/MMP9, Src and MEK/ERK signaling pathways and found that MMP9 
A

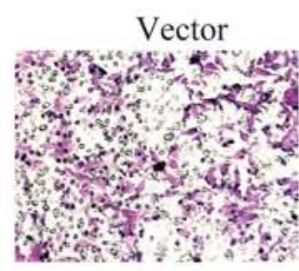

B

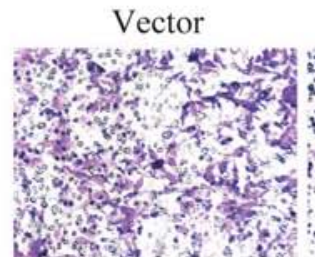

Sh-1

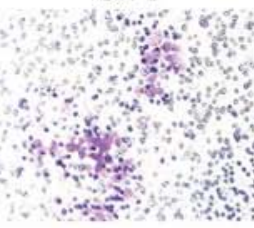

Sh-1 sh-2

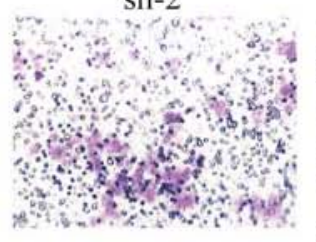

sh-2
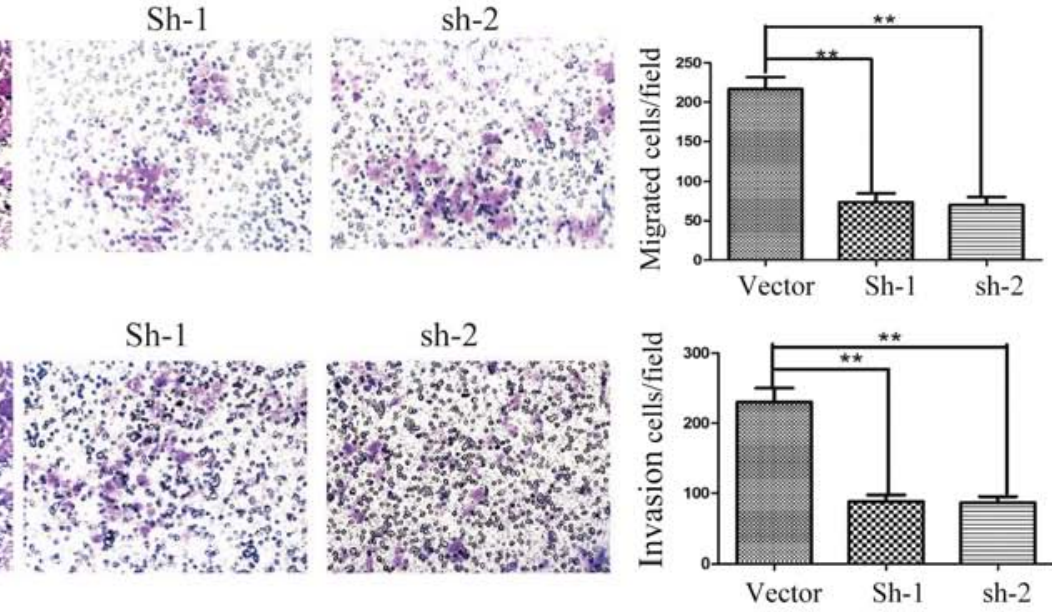

C

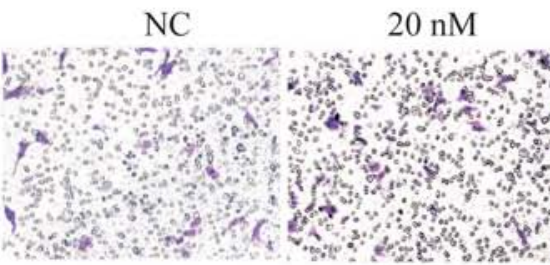

$50 \mathrm{nM}$
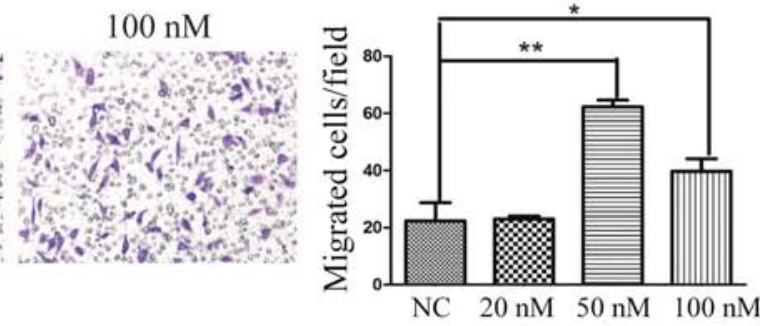

D

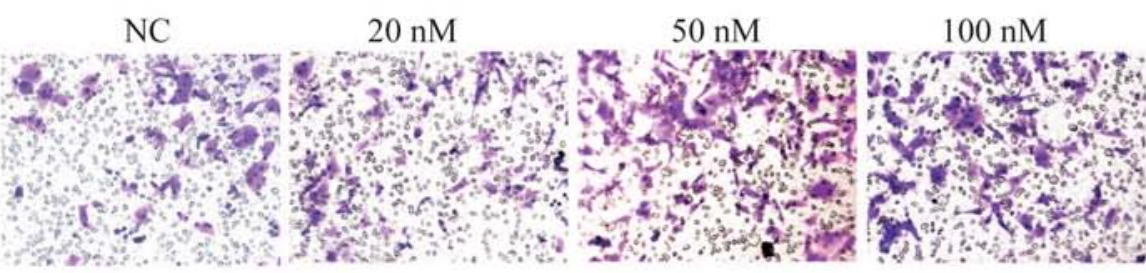

E
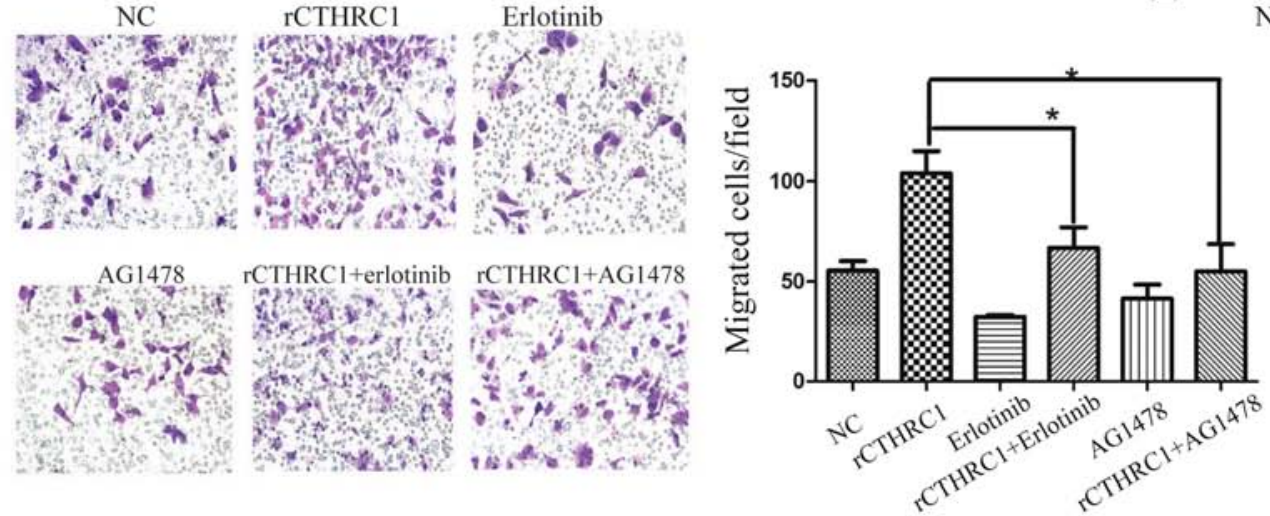

F

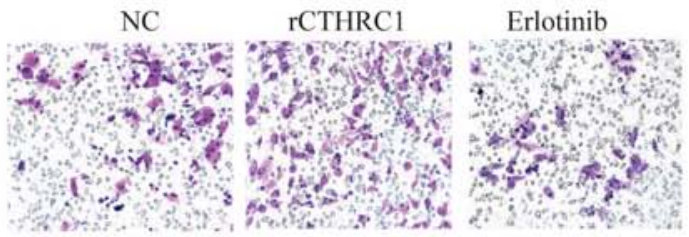

AG1478

rCTHRC1+erlotinib rCTHRC1+AG1478
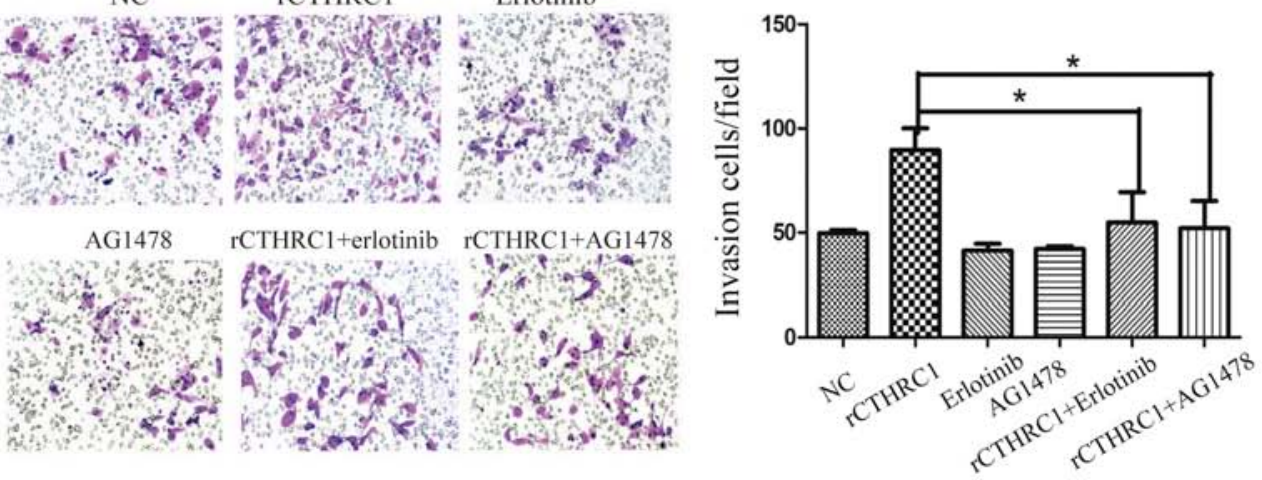

Figure 5. CTHRC1 promotes EOC cell invasion and migration in vitro. Transwell migration (A) and invasion (B) assays using ES2 cells transfected with Lenti-shCTHRC1-(1,2) or control vector. Transwell migration (C) and invasion (D) assays using SKOV3 cells treated with rCTHRC1 protein (at doses of 0, 20,50 and $100 \mathrm{nM}$ ) respectively. The pro-migration (E) and pro-invasion (F) effect of rCTHRC1 protein was partially blocked by AG1478/erlotinib as shown by Transwell assay in vitro. Representative images are shown on the left, and the quantification of 3 randomly selected fields is shown on the right. The results shown are mean $\pm \mathrm{SD}$ of migration or invading cells at $\mathrm{x} 200$ magnification per field. ${ }^{*} \mathrm{P}<0.05 ;{ }^{* *} \mathrm{P}<0.01$. 
A

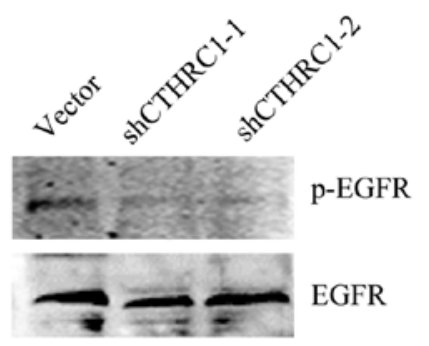

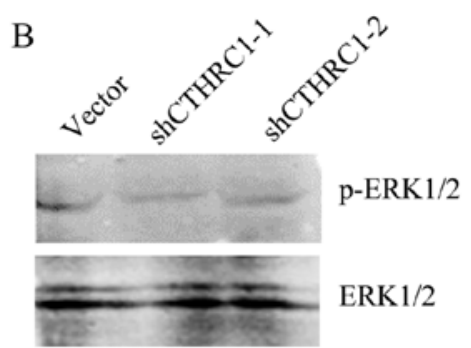
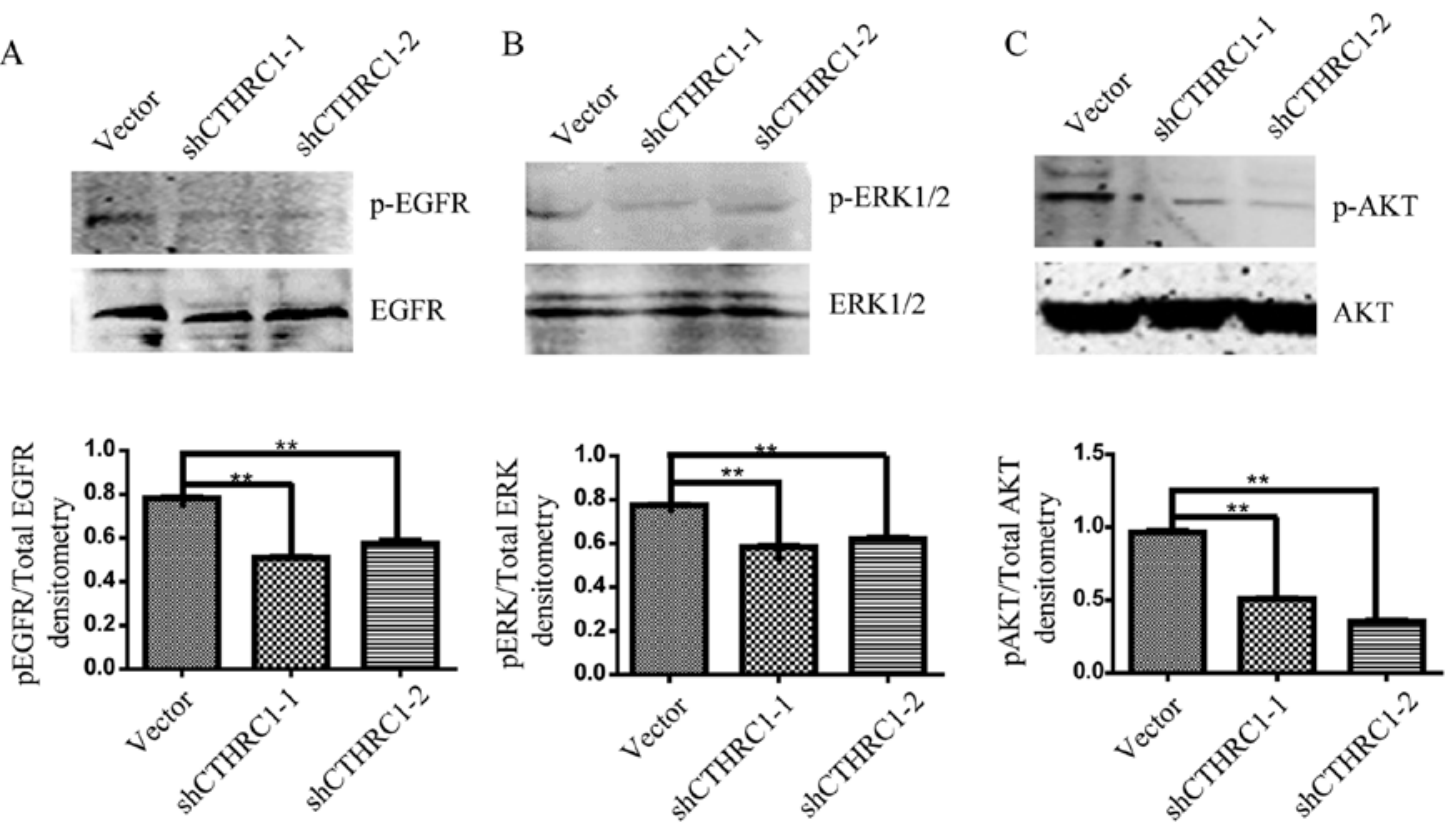

D

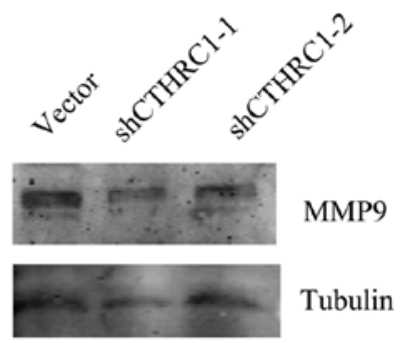

E
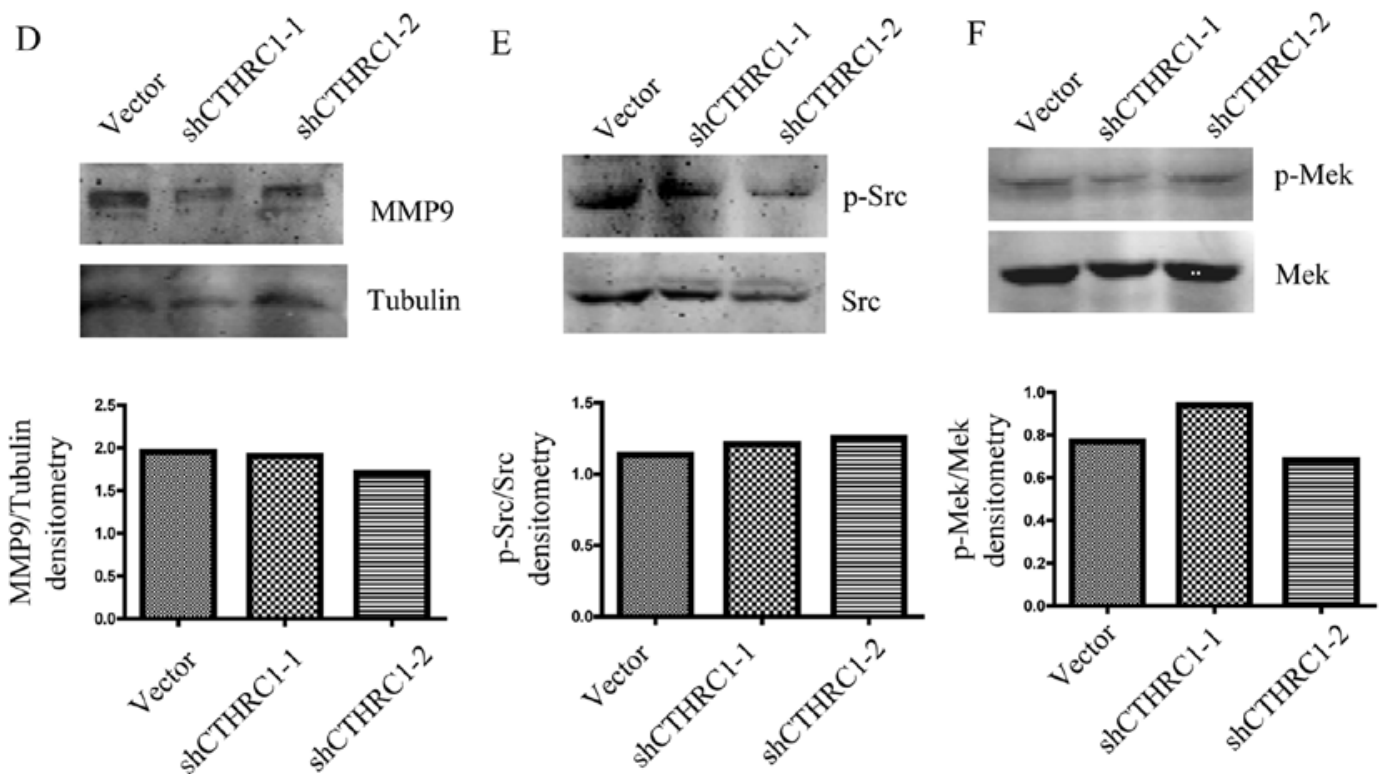

Figure 6. The expression of EGFR and phosphorylated EGFR (A), ERK1/2 and phosphorylated ERK1/2 (B), and AKT and phosphorylated AKT (C) in ES2 cells transfected with Lenti-shCTHRC1-(1,2) or control vector detected by western blotting. The expression of MMP9 (D), Src and phosphorylated Src (E), and MEK and phosphorylated MEK (F) in ES2 cells transfected with Lenti-shCTHRC1- $(1,2)$ or control vector detected by western blotting. Representative images are shown in the upper lane, and quantitative analysis of densitometry for p-ERK1/2/total EGFR, p-ERK1/2/total EGFR, p-AKT/total AKT, MMP9/ tubulin, p-Src/total Src and p-MEK/total MEK by ImageJ software are shown below. $\beta$-actin was used as an internal control. ${ }^{*} \mathrm{P}<0.05$, $^{* *} \mathrm{P}<0.01$.

expression and phosphorylated Src or MEK were not altered by CTHRC1 silencing (Fig. 6D-F).

Collectively, our data demonstrate that CTHRC1 is upregulated in human ovarian cancers and predicts poor prognosis. CTHRC1 functions as a pro-migration and pro-invasion factor in ovarian cancer, which is dependent on the EGFR/ERK1/2/ AKT signaling pathway.

\section{Discussion}

Metastasis remains the leading cause of relapse and death from ovarian cancer. Most women are diagnosed at the advanced stages of disease (FIGO stages III/IV) with disseminated intraperitoneal carcinomatosis (20). Although studies have identified components of signaling pathways whose aberrant expression and/or activity has been linked to ovarian cancer metastasis, the mechanisms are poorly understood. There is a critical need for better understanding of the molecular events that drive metastasis development and progression in ovarian cancer. CTHRC1 was found to be aberrantly upregulated in several malignant tumors, including melanoma, and cancers of the gastrointestinal tract, breast, thyroid, liver and the pancreas $(6,21)$.

Our study showed that the CTHRC1 was upregulated in malignant epithelial ovarian tumors and associated with FIGO stage, lymph node status and tumor size. Importantly, high 
CTHRC1 expression predicts poor survival of EOC patients, indicating that CTHRC1 is of value in EOC prognosis. It is noteworthy that the CTHRC1-positive rate is especially higher in advanced FIGO stage ovarian cancer patients, indicating that CTHRC1 expression may be involved in the metastasis of ovarian cancer. Further in vivo studies are needed to clarify whether CTHRC1 is a critical factor in EOC cancer metastasis.

Upregulation of CTHRC1 is a feature of many aggressive human cancers, being linked to tumor cell migration and invasion. In this study, we found that CTHRC1 silencing significantly inhibited migration and invasion of the ovarian cancer cell line ES2. Furthermore, as a secreted protein, recombinant CTHRC1 protein promoted ovarian cancer cell invasion in SKOV3. One possible reason why $50 \mathrm{nM}$ CTHRC1 was more efficient than $100 \mathrm{nM} \mathrm{CTHRC} 1$ is that $50 \mathrm{nM}$ may be the nearest concentration to CTHRC1 content in the ovarian cancer microenvironment and very high concentration of CTHRC1 may trigger different intracellular signaling pathways that counteracts the cancer cell invasion.

Although the functional roles of CTHRC1 in tumor cell invasion and metastasis have been well established, the underlying mechanisms of how CTHRC1 promotes cancer cell invasion is not fully understood. Several studies demonstrated that CTHRC1 can promote tumor cells migration and invasion by activating some signal pathways, such as Wnt/PCP-Rho, ERK/MMP9, Src/focal adhesion kinase and MEK/ERK signaling (22-24). In ovarian cancer cells, Hou et al demonstrated that CTHRC1 increased the invasive capabilities by activating the $\mathrm{Wnt} / \beta$-catenin signaling pathway (17). However, no report revealed the association of CTHRC1 with EGFR signaling.

Approximately $70 \%$ of epithelial ovarian cancer express activated EGFR (25). EGFR overexpression and activation result in increased proliferation and migration of solid tumors including ovarian cancer $(12,26,27)$. In this study, we showed that CTHRC1 promotes ovarian cancer cell invasion by activating EGFR signaling. The following evidence supported our finding. Western blotting showed that the phosphorylation of EGFR expression was significantly decreased after CTHRC1 knockdown, and the pro-invasion activity of the rCTHRC1 protein was blocked by inhibitors of EGFR. Existing data indicated that the effects of EGFR signaling on cell migration and invasion are mediated by PI3K/AKT and ERK1/2 signaling pathways in epithelial ovarian cancer $(14,28,29)$. In this study both expression of Akt and ERK1/2 phosphorylation were decreased after CTHRC1 knockdown in the same ovarian cancer cell line. Our results provide a novel mechanism mediated by CTHRC1 to induce ovarian cancer cell migration. Further deep mechanism investigations are needed to find a binding receptor of CTHRC1 in EOCs, a linker between secreted CTHRC1 expression and intracellular EGFR signaling activation.

Taken together, our results indicate CTHRC1 contribute to the progression of ovarian cancer through activating EGFR signaling and identified CTHRC1 as a potentially important molecule for human ovarian cancer prognosis. CTHRC1 together with EGFR can be considered as targets for treatment of epithelian ovarian cancer.

\section{Acknowledgements}

This study was supported by The Fifth People's Hospital of Shanghai Foundation (2015WYQJ03), Shanghai Key Laboratory of Female Reproductive Endocrine Related Diseases (14DZ2271700) and the National Natural Science Foundation of China (81101600).

\section{References}

1. Siegel RL, Miller KD and Jemal A: Cancer statistics, 2015. CA Cancer J Clin 65: 5-29, 2015.

2. Alonso DF, Ripoll GV, Garona J, Iannucci NB and Gomez DE: Metastasis: Recent discoveries and novel perioperative treatment strategies with particular interest in the hemostatic compound desmopressin. Curr Pharm Biotechnol 12: 1974-1980, 2011.

3. Rhim AD,MirekET,Aiello NM,Maitra A,Bailey JM,McAllisterF, Reichert M, Beatty GL, Rustgi AK, Vonderheide RH, et al: EMT and dissemination precede pancreatic tumor formation. Cell 148: 349-361, 2012.

4. Wu Z, Wu Z, Li J, Yang X, Wang Y, Yu Y, Ye J, Xu C, Qin W and $Z$ hang $Z$ : MCAM is a novel metastasis marker and regulates spreading, apoptosis and invasion of ovarian cancer cells. Tumour Biol 33: 1619-1628, 2012.

5. Durmus T, LeClair RJ, Park KS, Terzic A, Yoon JK and Lindner V: Expression analysis of the novel gene collagen triple helix repeat containing-1 (Cthrc1). Gene Expr Patterns 6: 935-940, 2006.

6. Tang L, Dai DL, Su M, Martinka M, Li G and Zhou Y: Aberrant expression of collagen triple helix repeat containing 1 in human solid cancers. Clin Cancer Res 12: 3716-3722, 2006.

7. Wang P, Wang YC, Chen XY, Shen ZY, Cao H, Zhang YJ, Yu J, Zhu JD, Lu YY and Fang JY: CTHRC1 is upregulated by promoter demethylation and transforming growth factor- $\beta 1$ and may be associated with metastasis in human gastric cancer. Cancer Sci 103: 1327-1333, 2012.

8. Ke Z, He W, Lai Y, Guo X, Chen S, Li S, Wang Y and Wang L: Overexpression of collagen triple helix repeat containing 1 (CTHRC1) is associated with tumour aggressiveness and poor prognosis in human non-small cell lung cancer. Oncotarget 5: 9410-9424, 2014.

9. Chan JK, Pham H, You XJ, Cloven NG, Burger RA, Rose GS, Van Nostrand K, Korc M, Disaia PJ and Fan H: Suppression of ovarian cancer cell tumorigenicity and evasion of cisplatin resistance using a truncated epidermal growth factor receptor in a rat model. Cancer Res 65: 3243-3248, 2005.

10. Bull Phelps SL, Schorge JO, Peyton MJ, Shigematsu H, Xiang LL, Miller DS and Lea JS: Implications of EGFR inhibition in ovarian cancer cell proliferation. Gynecol Oncol 109: 411-417, 2008.

11. Seshacharyulu P, Ponnusamy MP, Haridas D, Jain M, Ganti AK and Batra SK: Targeting the EGFR signaling pathway in cancer therapy. Expert Opin Ther Targets 16: 15-31, 2012.

12. Maihle NJ, Baron AT, Barrette BA, Boardman $\mathrm{CH}$, Christensen TA, Cora EM, Faupel-Badger JM, Greenwood T, Juneja SC, Lafky JM, et al: EGF/ErbB receptor family in ovarian cancer. Cancer Treat Res 107: 247-258, 2002.

13. Zhou C, Qiu L, Sun Y, Healey S, Wanebo H, Kouttab N, Di W, Yan B and Wan Y: Inhibition of EGFR/PI3K/AKT cell survival pathway promotes TSA's effect on cell death and migration in human ovarian cancer cells. Int J Oncol 29: 269-278, 2006.

14. Loganathan S, Kandala PK, Gupta P and Srivastava SK: Inhibition of EGFR-AKT axis results in the suppression of ovarian tumors in vitro and in preclinical mouse model. PLoS One 7: e43577, 2012.

15. Gan Y, Shi C, Inge L, Hibner M, Balducci J and Huang Y: Differential roles of ERK and Akt pathways in regulation of EGFR-mediated signaling and motility in prostate cancer cells. Oncogene 29: 4947-4958, 2010.

16. Hwang YP, Yun HJ, Choi JH, Han EH, Kim HG, Song GY, Kwon KI, Jeong TC and Jeong HG: Suppression of EGF-induced tumor cell migration and matrix metalloproteinase-9 expression by capsaicin via the inhibition of EGFR-mediated FAK/Akt, PKC/Raf/ERK, p38 MAPK, and AP-1 signaling. Mol Nutr Food Res 55: 594-605, 2011.

17. Hou M, Cheng Z, Shen H, He S, Li Y, Pan Y, Feng C, Chen X, Zhang Y, Lin M, et al: High expression of CTHRC1 promotes EMT of epithelial ovarian cancer (EOC) and is associated with poor prognosis. Oncotarget 6: 35813-35829, 2015. 
18. Zhou X, Hu Y, Dai L, Wang Y, Zhou J, Wang W, Di W and Qiu L: MicroRNA-7 inhibits tumor metastasis and reverses epithelialmesenchymal transition through AKT/ERK1/2 inactivation by targeting EGFR in epithelial ovarian cancer. PLoS One 9: e96718, 2014.

19. Cao C, Lu S, Sowa A, Kivlin R, Amaral A, Chu W, Yang H, Di W and Wan Y: Priming with EGFR tyrosine kinase inhibitor and EGF sensitizes ovarian cancer cells to respond to chemotherapeutical drugs. Cancer Lett 266: 249-262, 2008.

20. Khalil I, Brewer MA, Neyarapally T and Runowicz CD: The potential of biologic network models in understanding the etiopathogenesis of ovarian cancer. Gynecol Oncol 116: 282-285, 2010.

21. Kharaishvili G, Cizkova M, Bouchalova K, Mgebrishvili G, Kolar Z and Bouchal J: Collagen triple helix repeat containing 1 protein, periostin and versican in primary and metastatic breast cancer: An immunohistochemical study. J Clin Pathol 64: 977-982, 2011.

22. Ma MZ, Zhuang C, Yang XM, Zhang ZZ, Ma H, Zhang WM, You H, Qin W, Gu J, Yang S, et al: CTHRC1 acts as a prognostic factor and promotes invasiveness of gastrointestinal stromal tumors by activating Wnt/PCP-Rho signaling. Neoplasia 16: 265-278, 278.e1-278.e13, 2014.

23. Kim HC, Kim YS, Oh HW, Kim K, Oh SS, Kim JT, Kim BY, Lee SJ, Choe YK, Kim DH, et al: Collagen triple helix repeat containing 1 (CTHRC1) acts via ERK-dependent induction of MMP9 to promote invasion of colorectal cancer cells. Oncotarget 5: 519-529, 2014.
24. Park EH, Kim S, Jo JY, Kim SJ, Hwang Y, Kim JM, Song SY, Lee DK and Koh SS: Collagen triple helix repeat containing-1 promotes pancreatic cancer progression by regulating migration and adhesion of tumor cells. Carcinogenesis 34: 694-702, 2013.

25. Nicholson RI, Gee JM and Harper ME: EGFR and cancer prognosis. Eur J Cancer 37 (Suppl 4): S9-S15, 2001.

26. Han M, Liu M, Wang Y, Chen X, Xu J, Sun Y, Zhao L, Qu H, Fan Y and Wu C: Antagonism of miR-21 reverses epithelialmesenchymal transition and cancer stem cell phenotype through AKT/ERK1/2 inactivation by targeting PTEN. PLoS One 7: e39520, 2012.

27. Chang ZG, Wei JM, Qin CF, Hao K, Tian XD, Xie K, Xie XH and Yang YM: Suppression of the epidermal growth factor receptor inhibits epithelial-mesenchymal transition in human pancreatic cancer PANC-1 cells. Dig Dis Sci 57: 1181-1189, 2012.

28. Konishi H, Takagi A, Kurita A, Kaneda N and Matsuzaki T: PEGylated liposome IHL-305 markedly improved the survival of ovarian cancer peritoneal metastasis in mouse. BMC Cancer 12: 462, 2012.

29. Wittinger M, Vanhara P, El-Gazzar A, Savarese-Brenner B, Pils D, Anees M, Grunt TW, Sibilia M, Holcmann M, Horvat R, et al: $\mathrm{hVps} 37 \mathrm{~A}$ status affects prognosis and cetuximab sensitivity in ovarian cancer. Clin Cancer Res 17: 7816-7827, 2011. 